\title{
BMJ Open Analysing cardiovascular risk factors and related outcomes in a middle-aged to older adults population in Iran: a cohort protocol of the Shiraz Heart Study (SHS)
}

Mohammad Javad Zibaeenezhad, ${ }^{1}$ Haleh Ghaem, ${ }^{2}$ Nader Parsa, ${ }^{1}$ Mehrab Sayadi, ${ }^{1}$ Mehrdad Askarian, ${ }^{3}$ Mohammad Kasaei, ${ }^{1}$ Zahra Sohrabi, ${ }^{4}$ Azime Dehghani-Firouzabadi, ${ }^{5}$ Ali Nariman, ${ }^{1}$ Salma Radmanesh, ${ }^{6}$ Arya Mani, ${ }^{7}$ Ehsan Bahramali, ${ }^{8}$ Mohammad Hossein Nikoo, ${ }^{1}$ Ali Reza Moaref, ${ }^{1}$ Iman Razeghian-Jahromi ${ }^{\circ} 1$

To cite: Zibaeenezhad MJ, Ghaem $\mathrm{H}$, Parsa N, et al. Analysing cardiovascular risk factors and related outcomes in a middle-aged to older adults population in Iran: a cohort protocol of the Shiraz Heart Study (SHS). BMJ Open 2019;9:e026317. doi:10.1136/ bmjopen-2018-026317

- Prepublication history for this paper is available online. To view these files, please visit the journal online (http://dx.doi org/10.1136/bmjopen-2018026317).

Received 27 August 2018 Revised 7 January 2019 Accepted 31 January 2019

Check for updates

(C) Author(s) (or their employer(s)) 2019. Re-use permitted under CC BY-NC. No commercial re-use. See rights and permissions. Published by BMJ.

For numbered affiliations see end of article.

Correspondence to Dr Iman Razeghian-Jahromi; razejahromi@yahoo.com

\section{ABSTRACT}

Introduction The significant increase in the rate of morbidity and mortality due to cardiovascular diseases has become a health challenge globally. Lack of enough knowledge on the underlying causes in Iran and taking the unique characteristics of the Shiraz metropolitan city (the capital city of Fars Province) into consideration prompted us to conduct the Shiraz Heart Study. The aim of this study is to determine the predisposing elements leading to coronary heart disease, cerebrovascular disease and peripheral arterial disease.

Methods and analysis In this population-based, prospective study, family physician clinics will become the executive arms. Participants aged $40-70$ years old will be recruited to achieve a sample size of 10000. Socioeconomicta and anthropometric indices supplemented by physical activity, nutritional and psychological questionnaires, as well as routine blood laboratory tests, medical history and electrocardiographic records, will be collected at enrolment in clinics. In addition, blood samples will be obtained to explore the possible role of genetics in outcome occurrence. Follow-up with blood sampling, completion of a lifestyle questionnaire and evaluation of clinical risk factors will be carried out five times in a 2-year interval for all participants. Advanced statistical methods such as mixed model and time-to-event models will be used for data analysis.

Ethics and dissemination This study is in accordance with the Helsinki Declaration and has been approved by the Research Ethics Committee of Shiraz University of Medical Sciences (№: 2017-358). Signing a written informed consent is the preliminary step. Participants are free to withdraw on their request at any time. Collected data are kept encrypted in a software with authorities' access only. Findings of the study will be published at a national or international scale through peer-reviewed journals.

\section{Strengths and limitations of this study}

- This study will be the first cardiovascular-oriented cohort that will be carried out in a metropolitan city of Iran, which is a low-income/middle-income country from the unique region of the Middle East, with baseline data and five more times of data collection as follow-up.

- The Family Physician Program is not an established healthcare service throughout the country.

- With the logistics support of Shiraz University of Medical Sciences and employing hardware and software facilities that were prepared previously for the Family Physician Program, conducting the study will be easier in terms of practice.

- Considering the growing understanding about the influence of genes on the premature or unpredicted manifestations of cardiovascular disorders, this study will focus significantly on genetic investigations with the help of a diverse repertoire of genomes relevant to various ethnicities like Fars, Lur, and Ghashghaei (Turk) in Shiraz and also by relying on the created biobank.

- As this study is going to be conducted in a single city, the data may not exactly be generalisable to other towns and rural areas.

\section{BACKGROUND}

Cardiovascular diseases (CVDs) have been accounted for the highest share in all-cause mortality. ${ }^{1}$ As the public health standards are improved and the people age, chronic diseases such as CVDs become one of the main reasons for hospitalisation. This situation has imposed great burden on healthcare systems, and undoubtedly without applying targeted preventive strategies it will become worse. In a study conducted in Canada, 
one-tenth of individuals with one of the health problems such as hypertension, diabetes, heart disease or stroke encounter financial barriers, especially with respect to medications and having healthy food. Indeed, those with financial barriers experienced more disease-related hospitalisations and mortality than those who did not have these barriers. ${ }^{2}$

Hopefully, the rate of morbidity and mortality and the associated social and economic stress could be decreased by addressing modifiable risk factors. ${ }^{3}$ Obviously, identification of risk factors is the preliminary step. This began in the Framingham Heart Study in 1948. ${ }^{4}$ Although similar studies have been carried out throughout the world, it seems that the known risk factors cannot explain the entire incidence rate and the contemporary intriguing trend of cardiovascular events. Thus premature occurrence or unpredicted patterns of disease presentations remain a real challenge. ${ }^{5}$ Recent findings have shown that genetic factors play a prominent role. ${ }^{6}$ Moreover, evidence suggests that CVDs are determined by a complex interaction of genetic and environmental factors. ${ }^{5}$ If so, it should be speculated that the prevalence of associated risk factors is based on ethnicity and geographical areas. ${ }^{78}$

Over three-quarters of deaths due to CVDs occur in low-income and middle-income countries, ${ }^{1}$ and Iran is part of this group. Meanwhile, in the Middle East region, CVDs will be a considerable burden in the following years. ${ }^{9}$ Based on the last national census in 2016, about $75 \%$ of the Iranian population resides in urban areas. ${ }^{10}$ Also, most of the disability and mortality in this country result from coronary artery disease (CAD). ${ }^{11}$ Perhaps there is a link between living in Iranian urban areas and the high CAD rate. Lack of domestic data on cardiovascular risk factors is a matter of vacuity. Iran, as a low-income/middle-income country, has started investigations conducted in recent years.

The Shiraz Heart Study (SHS) will be the first cardiovascular-oriented cohort study in an Iranian metropolitan city. Shiraz, with a population of more than 1.7 million, ${ }^{10}$ is the capital of Fars Province and is geographically located south-west of Iran (figure 1).

It appears that urbanisation and the resultant changes in lifestyle endanger the health of the residents of Shiraz. Office-based and sedentary jobs are common among citizens. Women are employed in administrative jobs more than those in rural areas. The inherent nature of these jobs is accompanied by low physical activity and unhealthy nutritional habits. Anxiety levels and air pollution are more in comparison with small cities and towns. ${ }^{12-18}$ Moreover, a prominent feature of Shiraz is the existence of various ethnic groups, such as Fars, Lur, Ghashghaei (Turk) and others. ${ }^{19}$ This could be a good opportunity to elucidate the role of genetic differences in CVD occurrence or severity. ${ }^{20}$ Moreover, up to now there are genetic elements responsible for the concurrent incidence of cardiovascular risk factors, leading to what is known as the metabolic syndrome and its catastrophic consequences such as diabetes mellitus. ${ }^{21}$ Multiple other genetic links are yet to be elucidated.

\section{Aims}

The aim of this study is to assess the health status of Shiraz citizens with respect to the main vascular disorders and their related risk factors as among the major concerning issues responsible for hospitalisations and mortality.

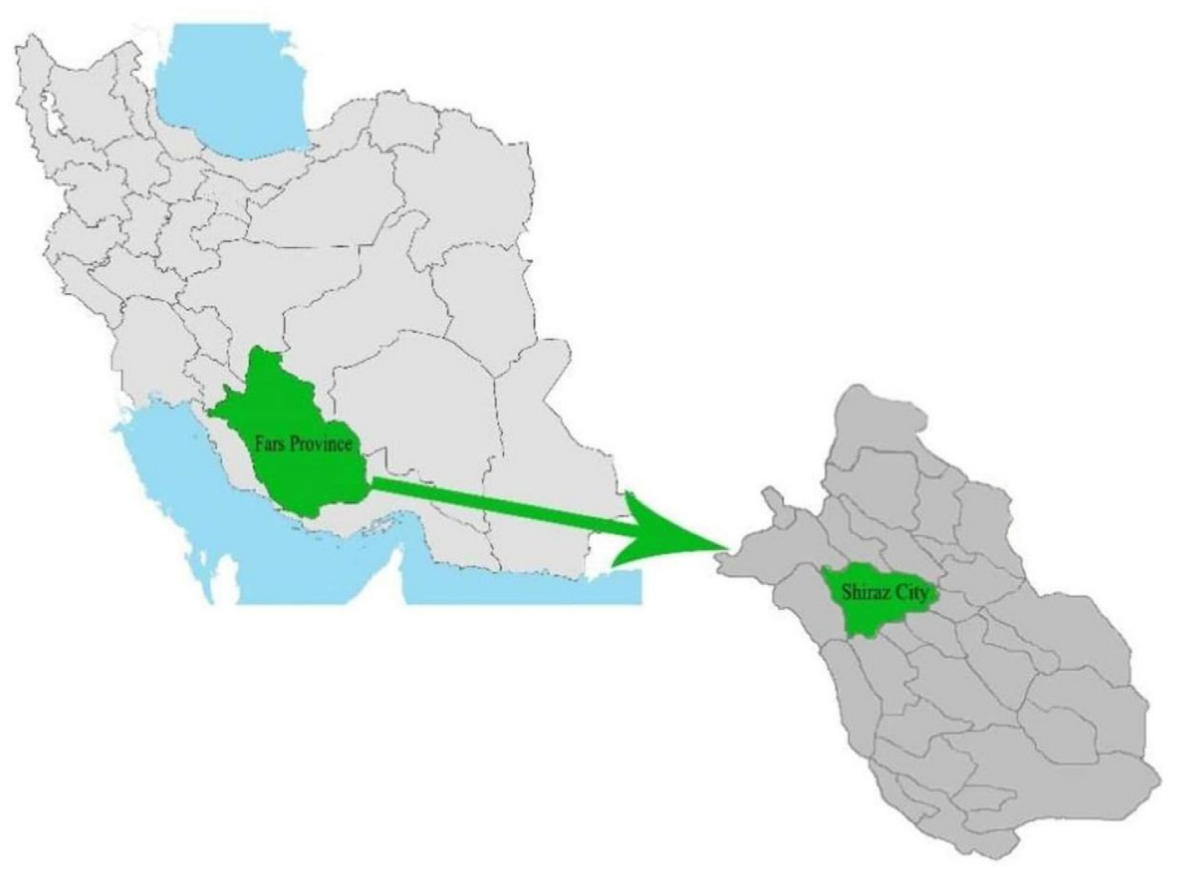

Figure 1 Location of Shiraz City, the capital of Fars Province in Iran. *Each participant should pass phase I in 1 day and phase II in another day. 
Using data extracted from this study, we hope that the rising trend of CVDs will be reduced by modifying health policies based on an evidence-based process. ${ }^{6}$ This research could also be an effective step forward in the prevention and control of cardiovascular events for the Iranian population and the rest of the world.

The following are the objectives:

- Clarify the prevalence of cardiovascular outcomes and their known risk factors at enrolment.

- Elucidate the incidence of cardiovascular outcomes, its related death and cardiovascular risk factors (known and possibly unknown) at follow-up.

- Identify lifestyle patterns such as nutritional habits, physical activity, smoking status, alcohol abuse and other relevant factors.

- Investigate the probable presence of non-modifiable cardiovascular risk factors with emphasis on the role of genes.

\section{METHODS AND ANALYSIS}

\section{Healthcare services in Shiraz}

The Family Physician Program is not an established healthcare service in all of Iran's provinces. The Iranian Ministry of Health selected Fars Province as one of the two pilot centres for this programme, and Shiraz as the main centre has started to prepare the required hardware and software facilities since 2012. In this structure, health services for prevention, diagnosis, treatment and rehabilitation have served the society in three distinct levels, namely general, specialty and subspecialty care. The first level, or the family physician level, includes a general physician who supervises a certain number of individuals around his/her own clinic region and is responsible for providing primary therapeutic service and recording each person's health status on dedicated files. A nurse or midwife attends to the family physician clinic to deliver relevant services. If a family physician makes a diagnosis and decides a patient needs to receive higher medical care, he/she is referred to the second level (specialist) or even the third level (subspecialist). The SHS is considered an independent programme in the family physician system and will be executed more easily, taking advantage of the established features.

\section{Study design}

In this study, family physician clinics are regarded as cohort study clusters. We divided Shiraz into north, south, east, west and central parts. Clinics were determined in each part, and then five of them were randomly selected. In the next step, the target population of each family physician clinic will be sorted based on age, and then will be selected through systematic sampling considering a sex ratio of 1:1. Family physicians have general and medical information on the relevant individuals that will be used in order to assign appropriate subjects. Considering the Shiraz population (1.7million) and the distribution of different socioeconomic levels throughout a wide area, multistage sampling is a suitable strategy with high coverage. In this setting, family physicians' enthusiasm to cooperate is important. Also, easy access and regular connection of participants to their relevant family physician could serve as a tool to encourage subjects to initiate and stay in the study. Clinics should be equipped with the necessary instrument for height measurement, digital scale for weight measurement, digital sphygmomanometer, blood sampling accessories and ECG machine. It is worth mentioning that all the mentioned tools should be the same in all clinics. In case of disparities, the cohort team is responsible for providing the tools. There is a possibility that the data collection steps will not be practical in a few family physician clinics due to limitations in physical environment or any other reasons. To address this potential problem, the cohort team will plan to have the respective participants attend another clinic to fulfil the steps.

\section{Study population}

About 2500 cases are covered per family physician, of whom around 700 people are between 40 and 70 years old. By assigning 25 clinics and 400 participants for each, a sample size of 10000 will be reached. Baseline enrolment may take 2 years to achieve. The inclusion criteria are residency in Shiraz urban areas at least for the past year from entry date and with no migration plan in the next few years. Moreover, some exclusion criteria are considered, including those living far from the study centres, those who have mental or physical disabilities incapable of cooperation and attending, and those who have documented outcomes of interest. If participants had a history of coronary artery bypass graft, percutaneous coronary intervention, positive angiographic reports or any valid evidence with respect to outcomes, they will be excluded from the study.

\section{Outcomes}

The outcomes are defined as coronary heart disease, cerebrovascular disease and peripheral arterial disease, and death following these events.

\section{Sample size calculation}

- Considering death as the rarest outcome and simultaneously according to the age limit for enrolment, the mortality rate is predicted to be $3 \%$ over a 10 -year period. This rate was predicted according to the reports released by the Iranian Ministry of Health.

- Compensating for the increased accuracy of cluster sampling method instead of simple random sampling and reducing the variance inflation factors, the effect of the design will be 1.5 .

- Given the relative error of $20 \%$ and $95 \%$ CI, the possibility of loss (non-response, missing sample) is about $30 \%$.

Considering the above criteria, the primary sample size was estimated at 6400 study subjects. Due to the essential of subgroup analysis of both genders, the primary sample size was multiplied by $50 \%(6400 \times 50 \%=3200)$. To maintain the minimum required sample for each gender, the final sample size will be made by adding 3200-6400, which 


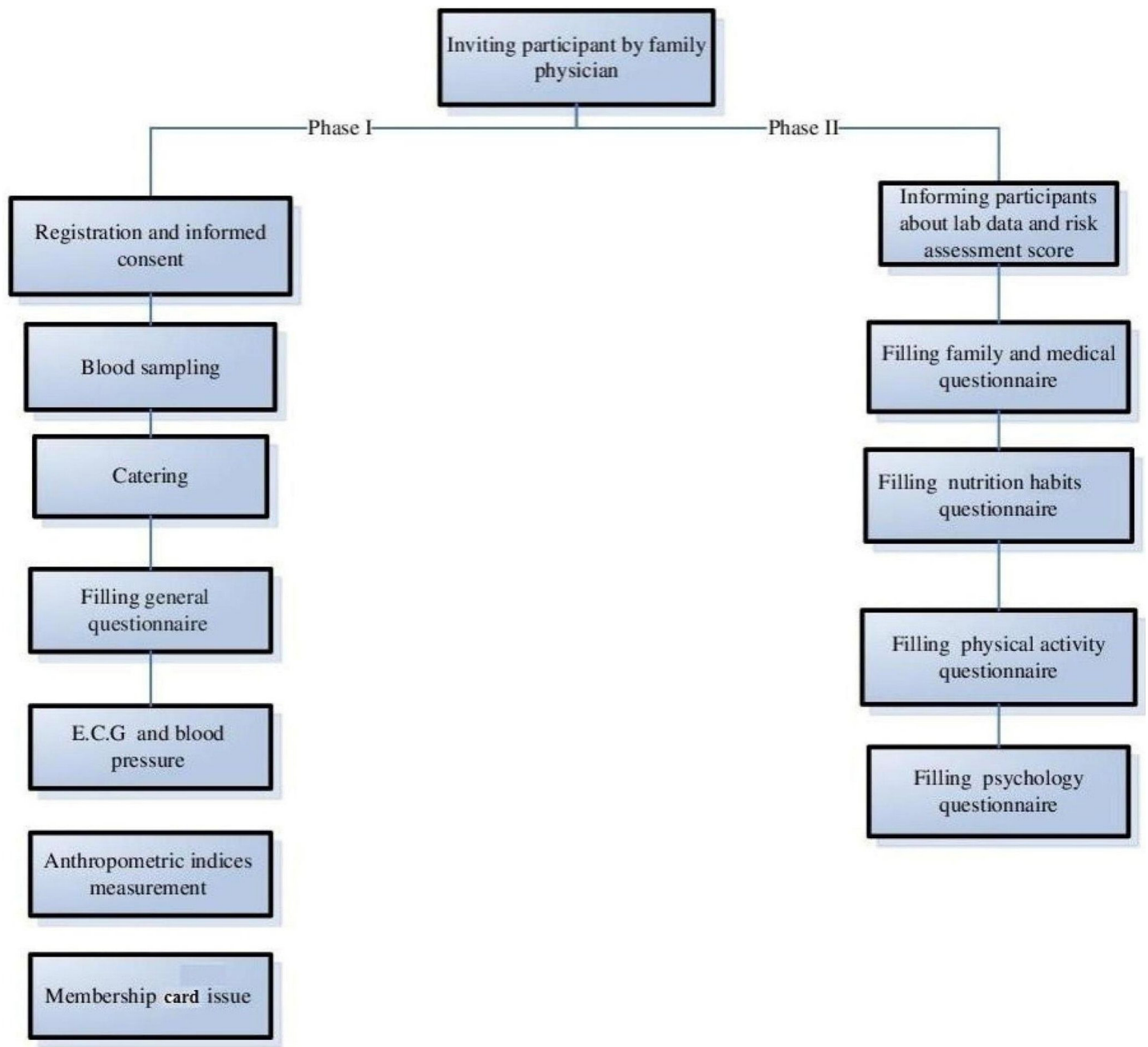

Figure 2 Schematic presentation of the enrolment process.

eventually will reach 9600 participants. This number was rounded up to 10000 as the intended goal.

\section{Recruitment}

The family physicians will be in charge of managing his/her own study group. The physician assistants will contact the subjects by telephone, and the project and its beneficial aspects will be explained to them. If they agree to participate, a verbal consent of contribution will be obtained, and a certain day and time will be arranged to attend the clinic. After reaching the target sample size in each clinic, the participants' list will be sent to the Cardiovascular Research Center (CRC). The cohort team will check and approve or request for substitution of the subjects based on the mentioned criteria.

\section{Data collection}

Measurements will be carried out during enrolment at baseline and five more times in a 2-year interval as follow-up. At baseline, the participants will pass the steps in two phases. Every phase will be carried out in 1 day (figure 2). Diverse data are going to be collected from the participants (table 1). All the questionnaires are provided electronically in a dedicated web-based software.
The family physicians, nurses or interviewers will be invited to the CRC and have a discussion meeting about the study procedures and decide to mutually cooperate. Family physicians individually sign the official contract for financial compensation for their time. If any of the family physicians at the beginning or during the first year of the study refuse to cooperate or withdraw for any reason, he/she will immediately be replaced by another one, and the latter is in charge of continuing the task for the enrolled participants. In the next step, a 2-day workshop training course will be held by the cohort team to transfer the necessary skills. Furthermore, periodic training will be considered for family physicians and other personnel during the study.

\section{Phase I}

Step 1: registration and obtaining informed consent

Participants should be fasting on the day of referral. The family physician will again explain the study process and how they will receive preventive health benefits. An informed consent will be signed by the study subject, and they will be assured that the enrolment is free of charge. An identification number will be assigned to each participant. This number, as a digital barcode, 
Table 1 Data collection using different methods at baseline

Different tools for collecting data

\begin{tabular}{|c|c|c|}
\hline Questionnaires & Blood sample & Instruments \\
\hline Psychological status questionnaire (GHQ) & $\begin{array}{l}\text { Biochemical parameters } \\
\text { (total cholesterol, HDL cholesterol, LDL } \\
\text { cholesterol, triglycerides, FBS, CR, BUN) }\end{array}$ & $\begin{array}{l}\text { Waist, hip and wrist circumference } \\
\text { (manually) }\end{array}$ \\
\hline
\end{tabular}

All questionnaires will be filled in through trained interviewers.

BUN, blood urea nitrogen; CBC, complete blood count; CR, creatinine; FBS, fasting blood sugar; FFQ, Food Frequency Questionnaire; GHQ, General Health Questionnaire; HDL, high-density lipoprotein; IPAQ, International Physical Activity Questionnaire; LDL, low-density lipoprotein.

is inserted in sampling accessories and is also shown on the questionnaires.

\section{Step 2: blood sampling}

Blood samples will be taken by a physician or nurse. A total of $17 \mathrm{~mL}$ is needed (table 2). A part $(5 \mathrm{~mL})$ would be sent on the same day in the cooler box to the laboratory of Shiraz Healthy Heart House centre for intended clinical tests. The identity of laboratory technician will be recorded as well for resolving any possible ambiguity. Other portions will be kept for future analysis. As an example, participation of individuals with CVDs from the same family from at least two generations will be predicted in the study. The existence of possible inherited traits will be sought. Moreover, investigating the genetic elements responsible for the concurrent incidence of CVD risk factors, single gene disorders, lethal genes, novel risk factor variants, rare variants and/or single nucleotide polymorphisms among congenital and acquired CVDs using a variety of techniques, including next-generation sequencing, whole exome sequencing and genome-wide association studies, could be carried out.

\section{Step 3: general questionnaire}

After catering, the participants will answer the general questions, which include personal information, sociodemographic data, ethnicity, kinship of parents, marital status, educational level, insurance type, occupation based on 15 different classifications, monthly income based on seven different categories and house ownership status.

\begin{tabular}{lll}
\hline \multicolumn{2}{l}{ Table 2} & Blood samples: quantity and purpose \\
\hline Number & Volume $(\mathbf{m L})$ & Purpose \\
\hline 1 & 5 & Blood tests \\
2 & 6 & Serum preparation \\
3 & 3 & DNA extraction \\
4 & 3 & Keeping the whole blood \\
\hline
\end{tabular}

\section{Step 4: recording blood pressure and ECG}

The participants will be asked to relax for $10 \mathrm{~min}$. Blood pressure of both arms will be measured consecutively (OMRON). After $10 \mathrm{~min}$, the measuring is repeated. This procedure is done for the third time, and the mean of the three measurements is recorded as the participant's blood pressure.

All clinics are equipped with similar 12-lead, computer-based digital ECG machine. Automatic measurement of relevant parameters, monitoring the vectors in two-dimensional/three-dimensional format, the possibility of ECG comparison between subjects and automatic diagnosis by its software (Cardiax) are among its capabilities. The physician evaluates the ECG. Moreover, they will be reviewed by the cardiologists to confirm the diagnosis, note down any required comments or request for repeat of the procedure.

\section{Step 5: measuring anthropometric indices}

Height, weight, and wrist, waist and hip circumference are the parameters that will be obtained in this step. Measurements are done based on similar training instructions received during the workshop of family physician assistants.

On completion of this phase, a membership card including information such as name, national identification, contact number, date of the next visit, phone numbers and address of the CRC and the probable existence of a specific disease will be given to the participants. If a participant is not willing to continue the study, he/ she is excluded and another matched one from the same centre will be randomly assigned.

\section{Phase II}

Step 1: informing the participants about the results of the first phase

In this session, the participants will be informed about the Framingham risk assessment, laboratory tests results, blood pressure and ECG. Any probable warning or need for higher medical consultation or other prescriptions 
will be given to them. This free health status screening provides a kind of encouragement for participants to commence and stay in the study.

\section{Step 2: family and medical questionnaire}

An interview in a private place will be held and data acquisition forms will be filled out with regard to history of CVDs, blood pressure, diabetes mellitus, hyperlipidaemia, hospitalisations and its documented causes besides smoking status, and drug and alcohol abuse, and subjects' sleeping pattern. Also, history of the probable existence of 13 other diseases, including asthma, allergy, chronic obstructive pulmonary disease, chronic renal disorder, chronic liver disorder, hypothyroidism or hyperthyroidism, goitre, rheumatology diseases, peptic ulcer, gastro-oesophageal reflux disease, gastritis and cancer, will be recorded. They will be asked to bring previous or current medications to maximise the precision of records. The medical history of first-degree relatives, particularly CVDs and related risk factors, will also be sought.

\section{Step 3: nutritional habits questionnaire}

The original framework of nutritional habits form was based on the Food Frequency Questionnaire with some modifications based on Iranian and Shiraz native food patterns. ${ }^{22}$ The eating habits of the participants are collected over a 1-year period on standard serving sizes. Information on daily energy and nutrient intake could be extracted.

\section{Step 4: physical activity questionnaire}

The International Physical Activity Questionnaire will be used to measure the physical activity of participants during the past 7 days. Activities in four different domains including in work, commuting, housework, leisure time and also sitting time are sought. ${ }^{23}$ The scores could be either in categories (low, moderate and high) or as continuous variables (metabolic equivalent task $[\mathrm{MET}]$ ).

\section{Step 5: psychology questionnaire}

The intended tool in this step is a 28-item version of the General Health Questionnaire. Based on mental health status, those with or at risk for psychiatric disorders will be determined. ${ }^{24}$ Its scores could be in continuous measures or Likert scale.

\section{Data management and analysis}

Assistants in the family physician clinics are responsible for supervision of filling in the questionnaires and for quality and quantity control of data entry. All the answers will be evaluated by epidemiology experts at the CRC in terms of accuracy of data entry and missing or unknown value on the same day. If items on the questionnaires are incomplete, they will be sent back to the centres, along with a written warning, and the clinic's assistants should check and correct them. Epidemiology experts will review and approve the data. Additionally, weekly data output will be checked to prevent any problems with regard to data recording, so that resolving in advance is feasible.
The CRC is the central office for management and coordination of the cohort programme. The cohort central team members are composed of scientific and executive experts. The scientific team or principal investigators include cardiologists, epidemiologists, pathologist, community physician, nutritionist, sport medicine doctor, geneticist and nurse. The interviewers will be randomly assessed by the cohort team members and receive the required proficiencies. Epidemiologists will specifically supervise the reliability of the whole procedures and will also be in charge of monitoring the implementation of the research process periodically.

All collected data at baseline, except outcomes, will be considered as exposures. Based on the occurrence of previously mentioned outcomes and proposals raised by the research committee of the SHS, different categories of analysis from conventional regression models for baseline data and mixed models and time-to-event analysis for follow-up data will be implemented. According to the type of exposure and outcome in the model, confounding, interaction or collinearity will be checked. Multiple imputation could be used to handle missing values. ${ }^{25-27}$

\section{The follow-up phase}

Family physicians oversee tracking of the study subjects in each centre. The follow-up team comprises a physician, a nurse and an interviewer. The nurse or interviewer makes a yearly phone call to participants. In case of no answer in a predetermined pattern, the follow-up team will track the participants through their relatives or goes to their postal address until the condition of the participant is clarified. Also, passive follow up will be used. In this regard, any change in the health status of study subjects will be collected, mainly through a family physician clinic. They permit the cohort team in the consent form to access medical data from any relevant centres, such as hospitals, laboratories or forensic organisations. If outcome(s) of interest is found, the individual will be invited to the clinic. After blood sampling, a predesigned lifestyle questionnaire, in addition to clinical risk factors, will be obtained. An outcome review team including three internists will assess and confirm the outcomes. Moreover, in a 2-year interval, participants enter the follow-up phases and the occurrence of previously mentioned outcomes will be sought (figure 3). Resampling and the questionnaire will also be considered.

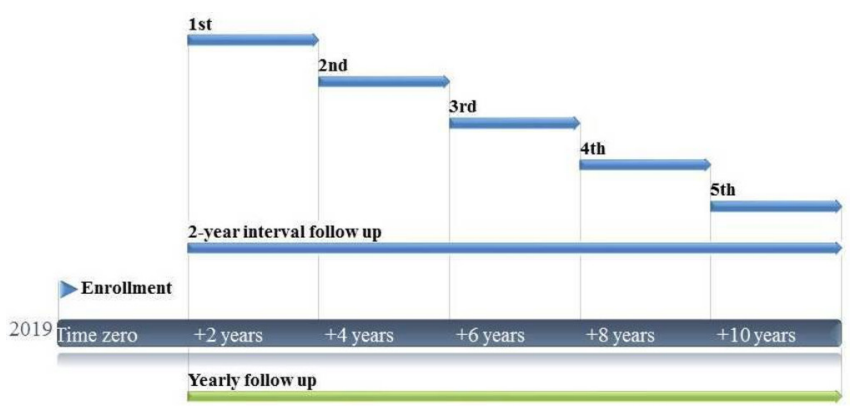

Figure 3 Timeline of enrolment and follow-up. 


\section{Patient and public involvement}

Participants were not involved in the development of research questions, outcome measures or in the design of the study. Also, they are not involved in the recruitment to or conduct of the study. The participants will be informed about their blood parameters, and the results of other examinations will also be gradually shared to them. The overall findings and benefits of the study will be disseminated through public media.

\section{DISCUSSION}

The SHS has been designed to fulfil important needs. All previous and current cohorts in Iran have been conducted in rural areas, or those carried out in urban regions did not focus on CVDs. One of the important aims of the present study is to determine the prevalence and incidence of the chief culprits of CVDs and their current risk factors. Consequently, correcting the direction of large-scale health policies is possible, and investments in this area can lead to the highest benefits applying preventive approaches.

Indeed, it would possibly uncover unknown risk factors. In recent years much effort has been made to find out the influence of genetic variations on cardiovascular disorders. The existence of two or more risk factors simultaneously in some individuals attracted investigators to search for the common reasons. It may be postulated that there are unique genes responsible for the concurrent appearance of risk factors such as dyslipidaemia, hypertension or obesity and what is known as metabolic syndrome. ${ }^{21}$

Due to accumulation of a vast variety of data, besides construction of a biobank, numerous fascinating national and international studies could be drawn and carried out. Also, this cohort acts as a training course for all involved personnel to share their acquired experiences in similar studies. The cohort team members hope that this study will help people in low-income and middle-income countries improve their life and welfare and move science some steps forward for the whole world.

\section{Author affiliations}

${ }^{1}$ Cardiovascular Research Center, Shiraz University of Medical Sciences, Shiraz, Iran (the Islamic Republic of)

${ }^{2}$ Research Center for Health Sciences, Institute of Health, Noncommunicable Diseases Research Center, Epidemiology Department, School of Health, Shiraz University of Medical Sciences, Shiraz, Iran

${ }^{3}$ Department of Community Medicine, School of Medicine, Medicinal and Natural Products Chemistry Research Center, Shiraz University of Medical Sciences, Shiraz, Iran (the Islamic Republic of)

${ }^{4}$ School of Nutrition and Food Sciences, Shiraz University of Medical Sciences, Shiraz, Iran (the Islamic Republic of)

${ }^{5}$ Sports Medicine Research Center, Cardiovascular Institute, Shiraz University of Medical Sciences, Shiraz, Iran (the Islamic Republic of)

${ }^{6}$ Department of Cardiovascular Medicine, School of Medicine, Shiraz University of Medical Sciences, Shiraz, Iran (the Islamic Republic of)

${ }^{7}$ Departments of Internal Medicine and Genetics, Yale University School of Medicine, New Haven, Connecticut, USA

${ }^{8}$ Noncommunicable Disease Research Center, Fasa University of Medical Sciences, Fasa, Iran (the Islamic Republic of)
Acknowledgements The cohort team would like to deeply appreciate the following: Mrs Zahra Daneshvar, Mrs Alamtaj Sanaei and Mr Jafar Zandi for their cooperation in the background, and also the technicians and computer experts of the CRC and the IT team members of Shiraz University under the leadership of Dr Amin Abbasi. Also, it is our duty to express our best feelings to the kind support provided by the Vice Chancellery of the SUMS.

Contributors MJZ contributed substantially to the concept and design of the study. $H G, N P, A A, E B$ and IR-J were responsible for study design as well as drafting of the paper. MS, MA, MK, ZS, AD-F, AN, SR, AM, MHN and ARM contributed to study design. All the authors revised the manuscript critically.

Funding This work was supported by the Vice Chancellery of Research Affairs of Shiraz University of Medical Sciences (grant number 93-7102). The funding source has no role in the study design and neither in the execution, interpretation, writing and results submission.

Competing interests None declared.

Patient consent for publication Not required.

Provenance and peer review Not commissioned; externally peer reviewed.

Open access This is an open access article distributed in accordance with the Creative Commons Attribution Non Commercial (CC BY-NC 4.0) license, which permits others to distribute, remix, adapt, build upon this work non-commercially, and license their derivative works on different terms, provided the original work is properly cited, appropriate credit is given, any changes made indicated, and the use is non-commercial. See: http://creativecommons.org/licenses/by-nc/4.0/.

\section{REFERENCES}

1. Organization, WH. Cardiovascular diseases (CVDs). Fact sheet: Organization, WH, 2017.

2. Campbell DJ, Manns BJ, Weaver RG, et al. Financial barriers and adverse clinical outcomes among patients with cardiovascularrelated chronic diseases: a cohort study. BMC Med 2017:15:33.

3. Zibaeenezhad MJ, Ostovan P, Mosavat SH, et al. Almond oil for patients with hyperlipidemia: A randomized open-label controlled clinical trial. Complement Ther Med 2019;42:33-6.

4. Long MT, Fox CS. The Framingham Heart Study--67 years of discovery in metabolic disease. Nat Rev Endocrinol 2016;12:177-83.

5. O'Donnell CJ, Nabel EG. Genomics of cardiovascular disease. N Engl J Med 2011;365:2098-109.

6. Economou EK, Oikonomou E, Siasos G, et al. The role of microRNAs in coronary artery disease: From pathophysiology to diagnosis and treatment. Atherosclerosis 2015;241:624-33.

7. Najafi M, Sheikhvatan M. Gender differences in coronary artery disease: correlational study on dietary pattern and known cardiovascular risk factors. Int Cardiovasc Res J 2013;7:124.

8. Veghari G, Sedaghat M, Maghsodlo S, et al. Differences in the prevalence of obesity among fars-native, turkman, and sisstanish ethnic groups in Iranian Northern adults in 2010. Int Cardiovasc Res $J$ 2013;7:56.

9. Motlagh B, O'Donnell M, Yusuf S. Prevalence of cardiovascular risk factors in the Middle East: a systematic review. Eur J Cardiovasc Prev Rehabil 2009;16:268-80.

10. Amar.org. National population and housing census. $2016 \mathrm{https} / / /$ www.amar.org.ir/.

11. Hatmi ZN, Tahvildari S, Gafarzadeh Motlag A, et al. Prevalence of coronary artery disease risk factors in Iran: a population based survey. BMC Cardiovasc Disord 2007;7:32.

12. Azak A, Huddam B, Gonen N, et al. Salt intake is associated with inflammation in chronic heart failure. Int Cardiovasc Res $J$ 2014;8:89.

13. Delisle H, Ntandou-Bouzitou G, Agueh V, et al. Urbanisation, nutrition transition and cardiometabolic risk: the Benin study. Br J Nutr 2012;107:1534-44.

14. Esfandiari-Baiat M, et al. Urbanisation and Its Effects on Water, Food Security and Energy Needs in Iran: a case study of city of shiraz, in the security of water, food, energy and liveability of Cities. Iran: Springer, 2014:101-12.

15. Firuzi O, Shakibazad N, Amoozgar H, et al. Effects of omega-3 polyunsaturated Fatty acids on heart function and oxidative stress biomarkers in pediatric patients with dilated cardiomyopathy. Int Cardiovasc Res J 2013;7:8.

16. Hassanzadeh-Rostami Z, Kavosi E, Nasihatkon A. Overweight and obesity among preschool children from fars province of iran: prevalence and associated factors. J Res Health Sci 2016;16:26-30.

17. Zamiriam M, et al. Correlation between HDL-C and smoking in teachers residing in Shiraz, Iran. International Cardivascular Research Journal 2011;5:61-5. 
18. Zibaeenezhad MJ, et al. Systolic and diastolic blood pressure among three groups of occupation. International Cardivascular Research Journal 2011;5:87-91.

19. Nosy Tourist. Iranian ethnicities. 2017 http://nosytourist.com/ iranethnicities.

20. Roberts R, Marian AJ, Dandona S, et al. Genomics in cardiovascular disease. J Am Coll Cardiol 2013;61:2029-37.

21. Keramati AR, Fathzadeh M, Go GW, et al. A form of the metabolic syndrome associated with mutations in DYRK1B. N Engl J Med 2014;370:1909-19.

22. Farjam M, Bahrami H, Bahramali E, et al. A cohort study protocol to analyze the predisposing factors to common chronic noncommunicable diseases in rural areas: fasa cohort study. BMC Public Health 2016;16:1090.

23. Moghaddam MB, et al. The Iranian Version of International Physical Activity Questionnaire (IPAQ) in Iran: content and construct validity, factor structure, internal consistency and stability. World App/ Sci 2012;18:1073-80.

24. Noorbala A, Mohammad K. The validation of general health questionnaire-28 as a psychiatric screening tool. Hakim Research Journal 2009;11:47-53.

25. Ghaem Maralani H, Tai BC, Wong TY, et al. Metabolic syndrome and mortality in the elderly: a time-dependent association. Diabetes Res Clin Pract 2013;99:209-16.

26. Ghaem Maralani H, Tai BC, Wong TY, et al. Metabolic syndrome and risk of age-related cataract over time: an analysis of intervalcensored data using a random-effects model. Invest Ophthalmol Vis Sci 2013;54:641-6.

27. Ghaem Maralani H, Tai BC, Wong TY, et al. The prognostic role of body mass index on mortality amongst the middle-aged and elderly: a competing risk analysis. Diabetes Res Clin Pract 2014;103:42-50. 